\title{
A Very Fast Three-Dimensional Impurity Profile Simulation Incorporating An Accumulated Diffusion Length and Its Application to the Design of Power MOSFETs
}

\author{
Shiroo Kamohara ${ }^{a}$, Masahiro Sugaya ${ }^{a}$, Hitoshi Matsuo ${ }^{b}$ \\ aSemiconductor Development Center, \\ Semiconductor \& Integrated Circuit Div., Hitachi, Ltd., \\ 1-280, Higashi-koigakubo Kokubunji, Tokyo 185, Japan \\ bCentral Research Laboratory, Hitachi, Ltd., \\ 1-280, Higashi-koigakubo Kokubunji, Tokyo 185, Japan
}

\begin{abstract}
In this paper, we introduce a method for the fast simulation of 3D impurity profile simulation. We analytically integrated the approximated non-linear transport equation and derived an analytical equation of 3D non-linear diffusion by introducing a new physical parameter, called the Accumulated Diffusion Length (ADL). This method allows us to simulate a 3D-profile by using a coupled $1 D$ simulator and analytical equations, where the $A D L$ is obtained during $1 D$ simulation. Our new methodology is implemented in the 3D process simulator, SPIRITIII/ADL. Our 3D-TCAD system composed of SPIRIT-III/ADL and our in-house device simulator was applied to the design of 3D-shaped power MOSFETs.
\end{abstract}

\section{Introduction}

Technology CAD (TCAD) systems are becoming indispensable for predicting device performance for optimize process parameters before fabrication. While two-dimensional (2D) TCAD systems are widely used [1], three-dimensional (3D) systems have not yet become practical because of the great amount of CPU time needed. Some accelerated approaches to impurity profile simulation have been proposed [2,3]. Although NIM [2] which numerically integrates the non-linear impurity transport equation gives good accuracy, this approach does not make 3D simulation practical because of its insufficient CPU-time enhancement. FABRICS [3] which uses a coupled one-dimensional (1D) simulator and an expander with analytical equations can achieve more than 100 times enhancement of CPU time, but users must define the parameter which describes lateral diffusion. This parameter must be introduced empirically because the impurity diffusion is non-linear.

In this work, we analytically integrate the approximated non-linear transport equation and derive an analytical equation of 3D non-linear diffusion by introducing a new physical parameter, called the Accumulated Diffusion Length (ADL). The ADL is obtained during 1D simulation and does not need to be defined by users. This method allows us to simulate a 3Dprofile by using a coupled 1D simulator and analytical equations. Our new methodology is implemented in the 3D process simulator, SPIRIT-III/ADL. Our 3D-TCAD system composed of SPIRIT-III/ADL and our in-house device simulator CADDETH3 [4] was applied to the 
design of 3D-shaped power MOSFETs [5] and we confirmed that this TCAD system can be efficiently applied.

\section{Methodology of 3D Process Simulation}

By discretizing the time dependence, the impurity transport equation is expressed as

$$
\begin{aligned}
C_{n}\left(x_{n}, y_{n}, z_{n}\right)=\int_{-\infty}^{+\infty} \cdots \int_{-\infty}^{+\infty} d x_{n-1} \cdots d x_{0} d y_{n-1} \cdots d y_{0} d z_{n-1} \cdots d z_{0} \\
\quad x G_{n-1}^{x}\left(x_{n}-x_{n-1}\right) \cdots G_{n-1}^{y}\left(y_{n}-y_{n-1}\right) \cdots G_{n-1}^{z}\left(z_{n}-z_{n-1}\right) C_{0}\left(x_{0}, y_{0}, z_{0}\right)
\end{aligned}
$$

where $C$ is the impurity concentration, $\mathrm{G}$ is the Green's function, $\mathrm{C}_{0}$ is the initial impurity distribution, $\mathrm{x}$ and $\mathrm{y}$ are the lateral directions, and $\mathrm{z}$ is the depth from the surface. Here, subscripts indicate the time step number and superscripts indicate the direction of transport. Since we are simulating power MOSFETs with a planar structure, the Green's functions in the $\mathrm{x}$ and $\mathrm{y}$ directions are expressed by Gaussian functions. To make analytical integration in the $\mathrm{x}$ and $\mathrm{y}$ directions possible, we assume the diffusion length of the mesh points other than $\mathrm{x}_{\mathrm{i}}$ are identical to that of $\mathrm{x}_{\mathrm{i}}$ at each time step, as shown in Fig. 1. This approximation is reasonable because that for lateral integration within a small time step only a few mesh points around $\mathrm{x}_{\mathrm{i}}$ contribute to the concentration at $\mathrm{x}_{\mathrm{i}}$.

The Green's function has no explicit form in the $\mathrm{z}$ direction because of the structural asymmetry. However, we can replace integration in the $\mathrm{z}$ direction with $1 \mathrm{D}$-simulation. Thus, Eq. (1) reduces to

$$
C_{n}\left(x_{n}, y_{n}, z_{n}\right)=\varepsilon\left(x_{n}, y_{n}, z_{n}\right) f\left(z_{n}\right)
$$

where

$$
\begin{aligned}
\varepsilon\left(x_{n}, y_{n}, z_{n}\right)=\sum_{j} \frac{1}{2}\left(\operatorname{erf}\left(\frac{b_{j}-x_{n}}{\sqrt{2} \sigma_{n}\left(x_{n}, y_{n}, z_{n}\right)}\right)-\operatorname{erf}\left(\frac{a_{j}-x_{n}}{\sqrt{2} \sigma_{n}\left(x_{n}, y_{n}, z_{n}\right)}\right)\right) \\
\quad \times \frac{1}{2}\left(\operatorname{erf}\left(\frac{c_{j}-y_{n}}{\sqrt{2} \sigma_{n}\left(x_{n}, y_{n}, z_{n}\right)}\right)-\operatorname{erf}\left(\frac{d_{j}-y_{n}}{\sqrt{2} \sigma_{n}\left(x_{n}, y_{n}, z_{n}\right)}\right)\right),
\end{aligned}
$$

$\sigma_{\mathrm{n}}\left(\mathrm{x}_{\mathrm{n}}, \mathrm{y}_{\mathrm{n}}, \mathrm{z}_{\mathrm{n}}\right)^{2}=\sum_{\mathrm{k}=0}^{\mathrm{n}-1} \sigma_{\mathrm{k}}\left(\mathrm{x}_{\mathrm{k}}, \mathrm{y}_{\mathrm{k}}, \mathrm{z}_{\mathrm{k}}\right)^{2}+\sigma_{\perp}^{2}$,

$f$ is the result of one-dimensional (1D) simulation, the four points, $\left(a_{j}, c_{j}\right),\left(a_{j}, d_{j}\right),\left(b_{j}, d_{j}\right)$, $\left(b_{j}, c_{j}\right)$ shape the $j$-th rectangular region which is obtained by dividing the arbitrarily shaped mask patterns into rectangles. Here, $\sigma_{\mathrm{n}}$ is the ADL, where $\sigma_{\mathrm{k}}$ is the diffusion length at the $\mathrm{k}$ th time step and $\sigma_{\perp}$ is lateral standard deviation of the ion implantation. The ADL is obtained through 1D-simulation because at every time step in the numerical calculation, the 1Dsimulator calculates diffusion lengths at every mesh point. Then, the 1D distribution of the ADL is geometrically expanded to a 3D distribution. This approach is possible because the distribution of ADL values are almost symmetric below the rectangular region. In this algorithm, numerical simulations are performed in one dimensional only, and the 3D impurity 
distribution is calculated entirely by using the analytical form. To verify our approximation, we compare the impurity distributions for the $\mathrm{x}_{\mathrm{n}}$ direction obtained from a numerical simulation with those obtained using our method. As shown in Fig. 2, good agreement is obtained. Table 1 shows the CPU-time of SPIRIT-III/ADL. In Table 1, we also show the CPU-time of SPIRIT-I [6], our in-house 2D process simulator. CPU time was reduced by more than a factor of 100 in comparison with numerical analysis by SPIRIT-I.

\section{The 3D TCAD System and its Application to Power MOSFETs}

Figure 3 shows a 3D TCAD system composed of SPIRIT-III/ADL and CADDETH3. The SPIRIT-III/ADL includes the input data processor, the 3D-mesh generator, SPIRIT-1D [7], and an expander. The input processor reads the mask layout information and the process recipe, and automatically generates sub-masks whose regions all have the same process conditions. Several sets of input data for SPIRIT-1D are generated for each sub-mask. SPIRIT-1D simulations are performed to obtain a set of 1D results. The expander generates $3 \mathrm{D}$ impurity profiles using the $3 \mathrm{D}$ mesh and the set of $1 \mathrm{D}$ simulation results including the $1 \mathrm{D}$ distribution of the impurity and the ADL. The results are transferred to the $3 \mathrm{D}$ device simulator CADDETH-3. Since the process simulation is much faster, process model parameter calibration time was greatly reduced, making it possible to quickly obtain accurate simulation results at the device level.

Figure 4 (a) shows the 3D impurity profile obtained with SPIRIT-III/ADL. Figure 4(b) shows a comparison of the channel implantation dose versus Vth from the experiments and from simulations with our 3D TCAD system. Good agreement is obtained not only for the channel implantation dose versus Vth, but also for the drain voltage versus the drain current and the gate voltage versus the drain current.

\section{References}

[1] M. E. Lee et. al., IEEE Trans. on Computer-Aided Design, Vol. 7, pp. 181-189, 1988.

[2] X. Tian et al., Proc. NASECODE VI, pp. 540-545, 1989.

[3] S. R. Nassif et al., IEEE Trans. on Computer-Aided Design, Vol. 3, pp. 40-46, 1984.

[4] T. Toyabe et al., IEEE Trans. Electron Devices, ED-32,pp. 2038-2044, 1985.

[5] I. Yoshida et al., IEEE J. Solid State Circuits, Vol. SC-11, pp. 472-477, 1976.

[6] M. Ohgo et al.., IEEE Trans. on Computer-Aided Design, Vol. CAD-6, pp. 439-445, 1987.

[7] S. Kamohara et al., Proceedings of BCTM, pp.126-129, 1992. 


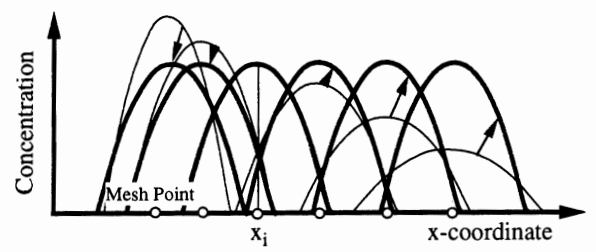

- Impurity Profile Expansion at Each Mesh Point

- Approximated Impurity Profile Expansion at Each Mesh Point Fig. 1. Approximation scheme.

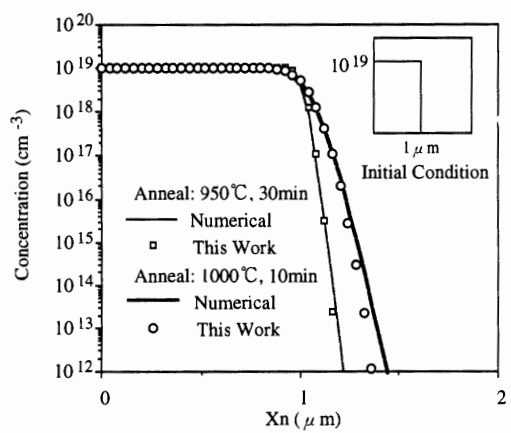

Fig. 2. Profile of $x$ direction comparison between the numerical approach and this work.

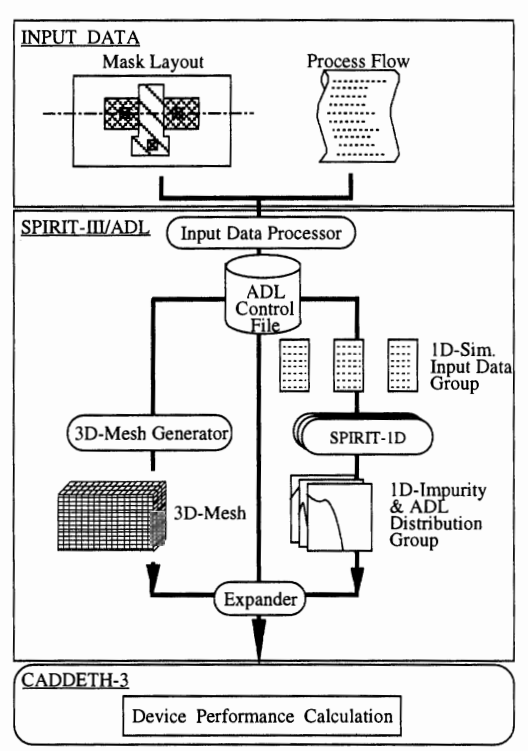

Fig. 3. Scheme of 3D-TCAD System.

Table 1. CPU-time of SPIRIT-III/ADL for some examples.

\begin{tabular}{ccccccc} 
& & & & \multicolumn{2}{c}{ CPU time* $(\mathrm{sec})$} \\
\cline { 5 - 6 } Example & Mesh Num. & Mask Num. & Dimensions & SPIRIT-IIUADL & SPIRIT-I [6] \\
\hline NMOS & 3491 & 6 & 2 & 23 & 6500 \\
PMOS & 3491 & 8 & 2 & 32 & 7000 \\
BJT & 3481 & 4 & 2 & 25 & 6300 \\
IGBT & 3481 & 5 & 2 & 38 & 7500 \\
Power-MOS & 61250 & 4 & 3 & 480 & --
\end{tabular}

* HITACHI: M-880 Mainframe Computer

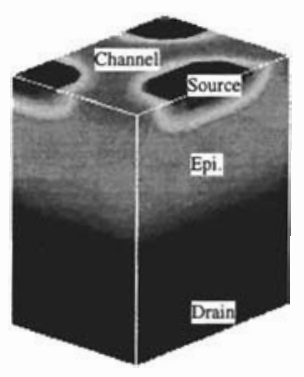

(a) Impurity Distribution of Power MOSFETs.

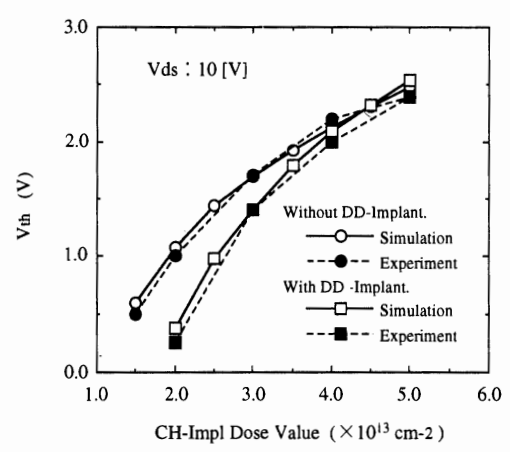

(b) Vth of Power MOSFETs.

Fig. 4. Application of 3D the TCAD system to Power MOSFET design. 